\title{
Relationship of Serum Total Bilirubin with Acute Coronary Syndromes at Sohag University Hospitals
}

\author{
Hassan Ahmad Hassanien, Yasser Mohamed Kamal, Mohamed Hussein Ahmed, Safaa Ali Ismail \\ Departments of Internal Medicine, Faculty of Medicine, Sohag University, Egypt \\ *Corresponding author: Safaa Ali Ismail, Mobile: (+20)1124304491, E-Mail: shmb49@yahoo.com
}

\begin{abstract}
Background: Many evidence has documented that the development of coronary artery disease involves lipid oxidation and formation of oxygen radicals as atherosclerosis and inflammation are associated with formation of oxygen and peroxyl radicals. Bilirubin has proven to be a potent antioxidant under physiological conditions by inhibiting both lipid and protein oxidation.

Objective: This study was aimed to show the relationship between serum total bilirubin and different types of acute coronary syndromes (STEMI, Non-STEMI, unstable angina) in patients admitted at Coronary Care Unit, Sohag University Hospital.

Patients and methods: This cross-sectional study included a total of 100 patients, 64 of them were men presented with acute coronary syndrome, admitted at Coronary Care Unit, Sohag University Hospital. This study was conducted between January 2020 to June 2020.

Results: The mean age was 55.4 years; 64 (64\%) were males, 44 (44\%) were current smokers, 37 (37\%) were HTN, 39 (39\%) were DM, 71 (71\%) had family history of IHD, 17 (17\%) were obese. When the patients were divided into 3 groups (group A, $\mathrm{n}=28$ "28\%", group B, $\mathrm{n}=13$ "13\%", and group $\mathbf{C}, \mathrm{n}=59$ "59\%"). Serum total bilirubin was significantly different (group A; Mean $\pm \mathrm{SD}=0.627 \pm 0.241$ ), (group B; Mean $\pm \mathrm{SD}=0.527 \pm 0.173$ ), (group C; Mean $\pm \mathrm{SD}=0.795 \pm 0.367)$. "P-value $=0.007$; highly significant".

Conclusions: It could be concluded that there is an inverse relation of bilirubin with acute coronary syndromes (ACS). These strengths the fact that bilirubin acts as an antioxidant and has cardioprotective action and patients with ACS have lower levels of bilirubin.
\end{abstract}

\section{INTRODUCTION}

It is considered that more than half of cardiovascular disease (CVD) will be the largest cause of death and disability by 2020. Acute coronary syndrome, a common complication of coronary artery disease (CAD), considered the greatest proportion of $\operatorname{CVD}^{(1,2)}$.

Rupture of an atherosclerotic plaque in coronary artery is accused to be the cause of most cases of acute coronary syndromes (ACS), leading to formation of thrombus ${ }^{(3)}$.

Atherosclerosis which is resulting from over balance among radical generating, compared with radical scavenging systems, is called oxidative stress. Endothelial cells could be damaged directly or indirectly by many ways, which leads to accelerate the accumulation of atherogenic factors like LDL, leading to increase the permeability of the cell ${ }^{(4,5)}$.

Bilirubin is the end product of heme catabolism, which has 2 forms: indirect bilirubin (IDB) and direct bilirubin (DB). IDB is converted to DB in hepatic cells and excreted into bile ${ }^{\left({ }^{(}\right)}$. It was considered that bilirubin is a waste product at first, bilirubin now has been known to have antioxidant and anti-inflammatory properties based on the inhibition of endothelial adhesion molecules and antithrombotic effects ${ }^{(7)}$.

As bilirubin is a waste product of the heme oxygenase action, now considered to have strong relation with coronary artery lesion as it inhibit both lipid and protein oxidation leading to major antioxidant action. As little as small level of bilirubin $(10 \mathrm{nM})$, by rapid generation of bilirubin by biliverdin reductase, is found to be enough to protect cells against higher concentration of oxidants. In addition to antiinflammatory effects of bilirubin on vasculature by inhibition of vascular smooth muscle cells proliferation. So it is proved that bilirubin act against formation of the plaque and subsequent atherosclerosis ${ }^{(8)}$.

Bilirubin show anti-inflammatory effects on vasculature and inhibits proliferation of vascular smooth muscle cells. This has led to suggestions that mildly increased circulatory bilirubin may have a physiologic function to protect against disease processes that involve oxygen and peroxyl radicals or vice versa and many studies have shown relation with $\mathrm{CAD}^{(\boldsymbol{9}, \mathbf{1 0})}$.

Reduced levels of bilirubin were noted to be associated with higher prevalence of coronary artery disease considered as potential risk factor marker ${ }^{(\mathbf{1 1})}$.

This study was aimed to show the relationship between serum total bilirubin and different types of acute coronary syndromes (STEMI, Non STEMI, unstable angina) in patients admitted at Coronary Care Unit of Sohag University Hospital.

\section{PATIENT AND METHODS}

This cross-sectional study included a total of 100 patients presented with acute coronary syndrome, 
admitted at Coronary Care Unit, Sohag University Hospital. This study was conducted between January 2020 to June 2020.

Inclusion criteria: Patients with ACSs (non-ST segment elevation MI, ST segment elevation MI and unstable anginas) are selected on basis of history, examinations and investigations.

Exclusion Criteria: Non cardiac chest pain, hepatitis of any cause, hepatotoxic drugs: (Oral hypoglycaemics; Acarbose \& pioglitazone - Anti-seizure drugs; Carbamazepine - Anti-fungal drugs; Itraconazole \& ketoconazole - Anti-tuberculosis; Isoniazide), liver cirrhosis, recent major trauma, recent surgery, haemolytic jaundice, and obstruction of bile duct.

Patients mean age was 55.4 years, 64 of them were men and 36 were women. They were subjected to the following:

A. Full History:

- Risk factors (hypertension - diabetes hyperlipidaemia - smoking - family history of IHD and obesity).

- History of previous CCU admission.

- History of previous ACS.

- History of cardiac catheterization.

- History of liver disease.

B. Examination:

- Vital signs (Pulse, Blood pressure, Temperature \& Respiratory rate).

- General examination (body mass index and detection of jaundice).

- Systemic examination (cardiac examination, abdominal examination and chest examination).

C. Investigations:

- $\quad$ ECG.

- Abdominal U/S.

- Cardiac enzymes: troponin \& CK-MB.

- Lipogram: total cholesterol, triglycerides, LDL \& HDL.

- Blood sugar: random blood glucose.

- $\mathrm{CBC}$.

- Liver Function tests (ALT, AST total bilirubin, direct bilirubin, indirect bilirubin, PT, PC and INR).

- Hepatitis markers: anti HCV \& anti HBV.

According to results of history, examination, ECG and cardiac enzymes patients were divided into three groups:

1. Group (A); Patients with STEMI (an event in which transmural myocardial ischemia results in myocardial injury or necrosis) ${ }^{(\mathbf{1 2})}$.

2. Group (B); Patients with Non-STEMI (considered the intermediate form of ACS, blockage either occurs in a minor coronary artery or causes partial obstruction of a major coronary artery) ${ }^{(\mathbf{1 3})}$.

3. Group (C); Patients with unstable (type of ACS in which there is myocardial ischemia without detectable myocardial necrosis) ${ }^{(\mathbf{1 4})}$.

The serum total bilirubin was compared in the three studied groups.

Ethical consent:

An approval of the study was obtained from Sohag University Academic and Ethical Committee. Every patient signed an informed written consent for acceptance of the operation. This work has been carried out in accordance with The Code of Ethics of the World Medical Association (Declaration of Helsinki) for studies involving humans.

\section{Statistical analysis}

All data were collected and analyzed using Statistical Package for Social Science (SPSS V20) to detect relationship of serum total bilirubin with acute coronary syndromes (ACSs). Data expressed as mean, standard deviation (SD), number and percentage. Mean and standard deviation were used as descriptive value for quantitative data, while number and percentage were used to describe qualitative data.

Chi-Square test was used for comparison between qualitative variables. Quantitative data was tested for normality by Shapiro-Wilk test. The nonparametric Mann-Whitney U test was used for comparing two quantitative variables. Kruskal-Wallis H test was used for comparison between more than two quantitative variables when variables were non parametric. Student $\mathbf{t}$ test was used to compare the means between two groups, and one-way analysis of variance (ANOVA) test was used to compare means of more than two groups. Mann Whitney test was used instead of Student $t$ test in case of non-parametric data. Tukey's range test is a single step multiple comparison procedure and statistical test. It can be used to find means that are significantly different from each other. Pearson Chi square was used to compare percentages of qualitative data, and Fisher's Exact test was used for non-parametric data. Pearson correlation test was used to compare two quantitative variables. P-value is considered statistically significant if $<0.05$, highly significant if $<0.001 \&$ non-significant if $\geq 0.05$

\section{RESULTS}

The mean age was $55.4 \pm 9.622$ years; $64(64 \%)$ were males, $44(44 \%)$ were current smokers, $37(37 \%)$ were HTN, 39 (39\%) were DM, $71(71 \%)$ had family history of IHD, $17(17 \%)$ were obese. When the patients were divided into 3 groups (group $\mathbf{A}, \mathrm{n}=28$ "28\%", group $\mathbf{B}, \mathrm{n}=13$ "13\%", group $\mathbf{C}, \mathrm{n}=59 " 59 \% ")$, the serum total bilirubin was significantly different (group $\mathrm{A}$; Mean $\pm \mathrm{SD}=0.627 \pm 0.241$ ), (group B; Mean \pm SD $=0.527 \pm 0.173),($ group $C ;$ Mean $\pm \mathrm{SD}=0.795 \pm$ $0.367)$. "P-value $=0.007$; highly significant" Table (1). 
Table (1): Patient's characteristics

\begin{tabular}{|c|c|c|c|c|c|}
\hline \multicolumn{2}{|c|}{ Group } & $\begin{array}{c}\mathbf{A} \\
(\mathrm{N}=28)\end{array}$ & $\begin{array}{c}\mathbf{B} \\
(\mathrm{N}=13)\end{array}$ & $\begin{array}{c}\mathrm{C} \\
(\mathrm{N}=59)\end{array}$ & P-value \\
\hline \multicolumn{2}{|c|}{$\begin{array}{l}\text { Age (years) } \\
(\text { Mean } \pm \text { SD) }\end{array}$} & $57 \pm 3.349$ & $59.615 \pm 2.839$ & $53.712 \pm 1.108$ & $\begin{array}{c}\mathbf{0 . 0 7 7} \\
\text { NS }\end{array}$ \\
\hline \multirow{2}{*}{$\begin{array}{l}\text { Gender } \\
\text { (Number) }\end{array}$} & Males & $\begin{array}{c}16 \\
(57.14 \%) \\
\end{array}$ & $\begin{array}{c}7 \\
(53.85 \%) \\
\end{array}$ & $\begin{array}{c}41 \\
(69.49 \%) \\
\end{array}$ & \multirow{2}{*}{$\begin{array}{c}\mathbf{0 . 3 8 2} \\
\text { NS }\end{array}$} \\
\hline & Females & $\begin{array}{c}12 \\
(42.86 \%)\end{array}$ & $\begin{array}{c}6 \\
(46.15 \%)\end{array}$ & $\begin{array}{c}18 \\
(30.51 \%)\end{array}$ & \\
\hline \multicolumn{2}{|c|}{ HTN (Number) } & $\begin{array}{c}3 \\
(10.71 \%)\end{array}$ & $\begin{array}{c}6 \\
(46.15 \%)\end{array}$ & $\begin{array}{c}28 \\
(47.46 \%)\end{array}$ & $\begin{array}{c}\mathbf{0 . 0 0 3} \\
S\end{array}$ \\
\hline \multicolumn{2}{|c|}{ DM (Number) } & $\begin{array}{c}11 \\
(39.29 \%) \\
\end{array}$ & $\begin{array}{c}6 \\
(46.15 \%) \\
\end{array}$ & $\begin{array}{c}22 \\
(37.29 \%) \\
\end{array}$ & $\begin{array}{c}\mathbf{0 . 8 3 8} \\
\mathrm{NS} \\
\end{array}$ \\
\hline \multicolumn{2}{|c|}{ Family History IHD } & $\begin{array}{c}22 \\
(78.57 \%)\end{array}$ & $\begin{array}{c}9 \\
(69.23 \%)\end{array}$ & $\begin{array}{c}40 \\
(67.79 \%)\end{array}$ & $\begin{array}{c}0.001 \\
S\end{array}$ \\
\hline \multicolumn{2}{|l|}{ Obesity } & $\begin{array}{c}6 \\
(21.42 \%) \\
\end{array}$ & $\begin{array}{c}2 \\
(15.38 \%) \\
\end{array}$ & $\begin{array}{c}9 \\
(15.25 \%) \\
\end{array}$ & $\begin{array}{c}\mathbf{0 . 2 8 4} \\
\mathrm{NS} \\
\end{array}$ \\
\hline \multicolumn{2}{|c|}{$\begin{array}{l}\text { BMI }(\mathbf{k g} / \mathbf{m 2}) \\
(M e a n \pm S D)\end{array}$} & $24.3 \pm 1.7$ & $25.1 \pm 1.5$ & $24.6 \pm 1.6$ & $\begin{array}{c}\mathbf{0 . 3 4 6} \\
\mathrm{NS} \\
\end{array}$ \\
\hline \multirow{3}{*}{$\begin{array}{l}\text { Smoking } \\
\text { (Number) }\end{array}$} & Smoker & $\begin{array}{c}12 \\
(42.85 \%)\end{array}$ & $\begin{array}{c}5 \\
(38.46 \%)\end{array}$ & $\begin{array}{c}27 \\
(45.76 \%)\end{array}$ & \multirow{3}{*}{$\begin{array}{c}\mathbf{0 . 0 0 3} \\
\mathrm{S}\end{array}$} \\
\hline & $\begin{array}{l}\text { Non- } \\
\text { smoker }\end{array}$ & $\begin{array}{c}13 \\
(46.43 \%) \\
\end{array}$ & $\begin{array}{c}7 \\
(53.84 \%) \\
\end{array}$ & $\begin{array}{c}20 \\
(33.9 \%) \\
\end{array}$ & \\
\hline & $\begin{array}{l}\text { Ex- } \\
\text { smoker }\end{array}$ & $\begin{array}{c}3 \\
(10.72 \%) \\
\end{array}$ & $\begin{array}{c}1 \\
(7.7 \%)\end{array}$ & $\begin{array}{c}12 \\
(20.34 \%)\end{array}$ & \\
\hline
\end{tabular}

There were 28 patients (28\%) known to be STEMI and 13 patients (13\%) known to be NSTEMI and 59 patients (59\%) known to be unstable.

Table (2): Patient groups according to ECG \& cardiac enzymes.

\begin{tabular}{|c|c|c|}
\hline \hline \multicolumn{3}{|c|}{ ACS } \\
\hline \hline Group A & N & \% \\
\hline Group B & 28 & 28 \\
\hline Group C & 13 & 13 \\
\hline Total & 59 & 59 \\
\hline
\end{tabular}

In this study, the mean age was (57) years in group (A), (59.615) years in group (B), (53.712) years in group (C), (P value $=\mathbf{0 . 0 7 7}$, non-significant). There was non-significant difference between the three groups regarding age .

Table (3): Comparison between the studied groups regarding age.

\begin{tabular}{|c|c|c|c|c|c|}
\hline \multirow{2}{*}{ Age } & \multicolumn{3}{|c|}{ ACS } & \multicolumn{2}{|c|}{ ANOVA } \\
\hline & Group A & Group B & Group C & $\mathbf{F}$ & P-value \\
\hline Mean \pm SD & $\begin{array}{c}57.000 \\
\pm \\
3.349\end{array}$ & $\begin{array}{c}59.615 \\
\pm \\
3.839\end{array}$ & $\begin{array}{c}53.712 \\
\pm \\
1.108\end{array}$ & 2.626 & $\begin{array}{c}0.077 \\
\text { NS }\end{array}$ \\
\hline
\end{tabular}

In this study, group (A), 16 patients were males (57.14\%) \& 12 patients were females (42.86\%), in group (B) 7 patients were males $(53.85 \%) \& 6$ patients were females $(46.15 \%)$, in group $(\mathrm{C}), 41$ patients were males $(69.49 \%) \& 18$ patient were females $(30.51 \%),(\mathbf{P}$ value $=\mathbf{0 . 3 8 2}$ non-significant $)$. There was non-significant difference between the three groups regarding sex. 
Table (4): Comparison between the studied groups regarding gender.

\begin{tabular}{|c|c|c|c|c|c|c|c|c|c|c|}
\hline \multirow{3}{*}{ Gender } & \multicolumn{8}{|c|}{ ACS } & \multirow{2}{*}{\multicolumn{2}{|c|}{ Chi-Square }} \\
\hline & \multicolumn{2}{|c|}{ Group A } & \multicolumn{2}{|c|}{ Group B } & \multicolumn{2}{|c|}{ Group C } & \multicolumn{2}{|c|}{ Total } & & \\
\hline & $\mathbf{N}$ & $\%$ & $\mathbf{N}$ & $\%$ & $\mathbf{N}$ & $\%$ & $\mathbf{N}$ & $\%$ & $\overline{X^{2}}$ & P-value \\
\hline Male & 16 & 57.14 & 7 & 53.85 & 41 & 69.49 & 64 & 64 & & \\
\hline Female & 12 & 42.86 & 6 & 46.15 & 18 & 30.51 & 36 & 36 & 1.925 & 0.382 \\
\hline Total & 28 & 100 & 13 & 100 & 59 & 100 & 100 & 100 & & \\
\hline
\end{tabular}

*P-value is considered statistically significant if $<0.05$, highly significant if $<0.001 \&$ non-significant if $\geq 0.05$.

In this study, the mean BMI was (24.3) in group (A), (25.1) in group (B), (24.6) in group (C) $(\mathbf{P}$ value $=\mathbf{0 . 3 4 6}$, non-significant). There is non-significant difference between the three groups regarding BMI. In group (A), 6 patients were obese $(21.42 \%), 22$ patients were not obese (78.58\%), in group (B) 2 patients were obese $(15.38 \%) \& 11$ patients were not obese (84.62\%), in group (C), 9 patients were obese (15.25\%), 50 patients were not obese (84.75\%), (P value $=0.284$ Statistically Non-significant).

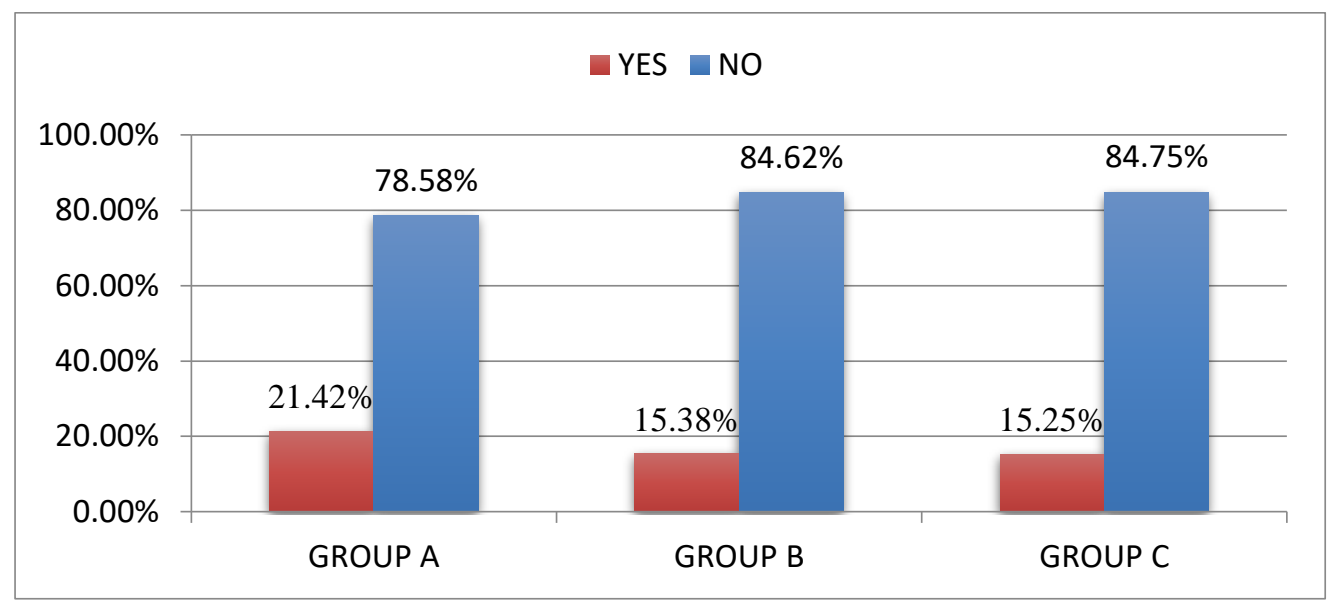

(P value $=0.284$ Statistically Non-significant $)$

Figure (1): Comparison between the studied groups regarding obesity.

In group (A), 12 patients were smokers $(42.85 \%), 3$ patients were ex-smokers $(10.72 \%) \& 13$ patients were nonsmokers (46.43\%), in group (B) 5 patients were smokers $(38.46 \%) \& 1$ patient was ex-smokers $(7.7 \%) \& 7$ patients were non-smokers (53.84\%), in group (C), 27 patients were smokers (45.76\%), 12 patients were ex-smokers $(20.34 \%)$ $\& 20$ patient was a non-smoker $(33.9 \%)$, (P value $=\mathbf{0 . 0 0 3}$ significant). There was significant difference between the three groups regarding smoking.

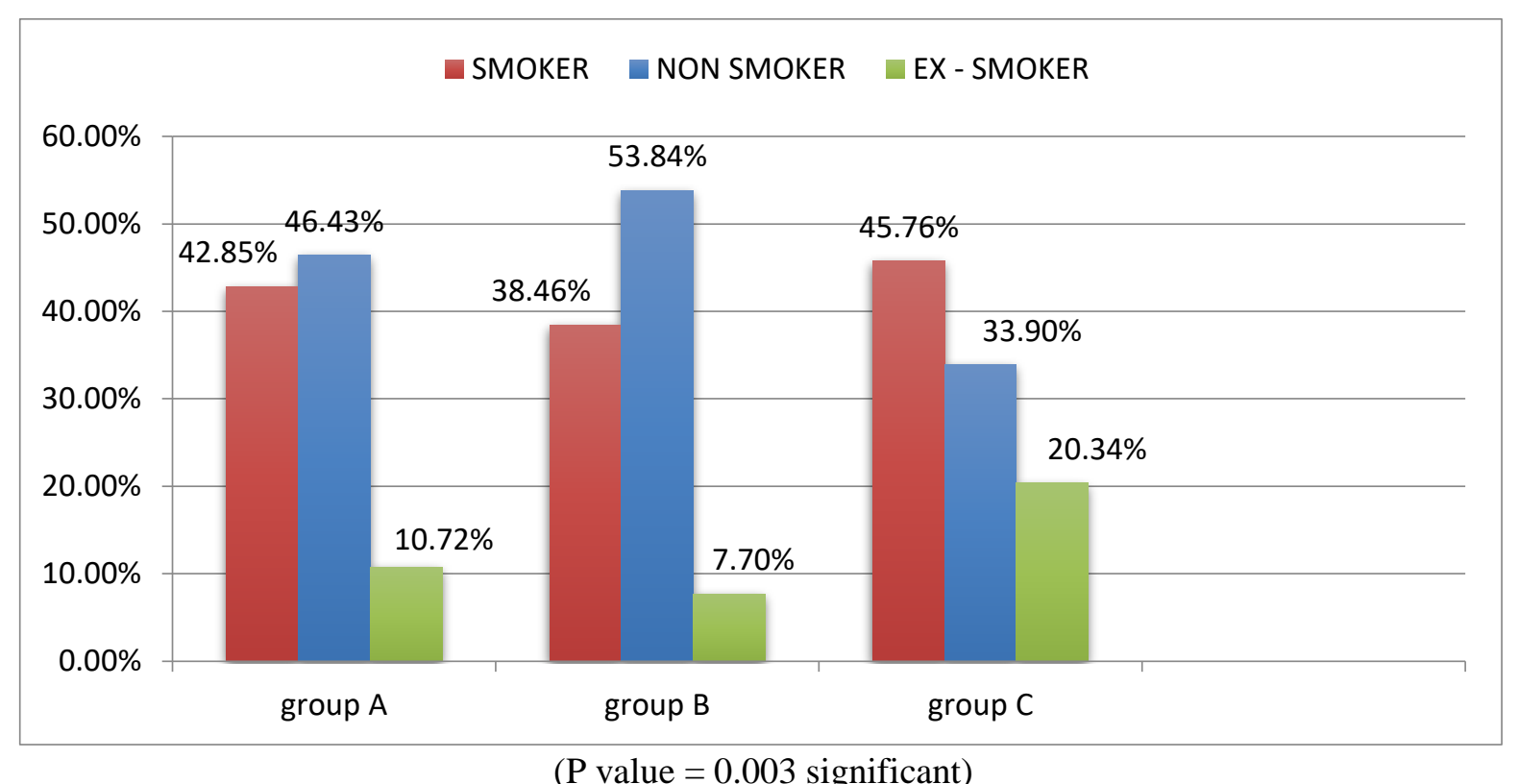

Figure (2): Comparison between the studied groups regarding smoking.

In group (A), 22 patients had family history IHD (78.57\%) \& 6 patients had not (21.43\%), in group (B) 9 patients had family history IHD (69.23\%) \& 4 patients had not (30.77\%), in group (C), 40 patients had family history IHD (67.79\%) $\& 19$ patients had not $(32.21 \%)$. (P value $=\mathbf{0 . 0 0 1}$ Statistically significant $)$. 


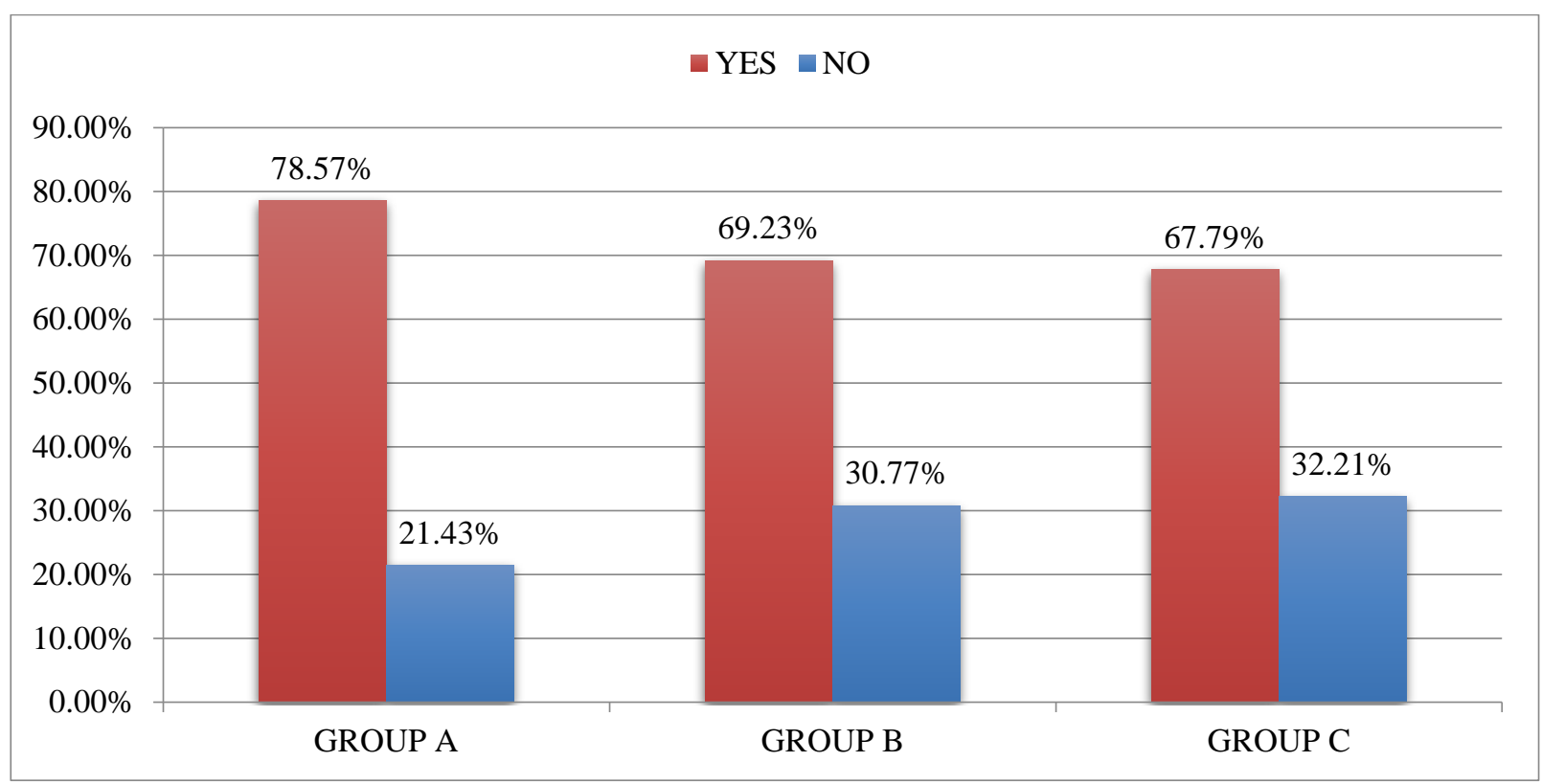

$(\mathrm{P}$ value $=0.001$ Statistically significant $)$

Figure (3): Comparison between the studied groups regarding family history of IHD.

In group (A), 3 patients were HTN (10.71\%), 25 patients were not HTN $(89.29 \%)$ \& 11 patients were DM (39.29\%) \& 17 patients were not DM (60.71\%), in group (B), 6 patients were HTN (46.15\%), 7 patients were not HTN $(53.85 \%)$ \& 6 patients were DM (46.15\%) \& 7 patients were not DM (53.85\%), in group (C), 28 patients were HTN (47.46\%), 31 patients were not HTN (52.54\%) \& 22 patients were DM (37.29\%) \& 37 patients were not DM (62.71\%) (P value for HTN = 0.003 statistically significant), (P value for $D M=0.838$ statistically non-significant $)$.

Table (5): Comparison between the studied groups regarding HTN \& DM

\begin{tabular}{|c|c|c|c|c|c|c|c|c|c|c|c|}
\hline & \multicolumn{8}{|c|}{ ACS } & \multirow{2}{*}{\multicolumn{2}{|c|}{ Chi-Square }} \\
\hline & & \multicolumn{2}{|c|}{ Group A } & \multicolumn{2}{|c|}{ Group B } & \multicolumn{2}{|c|}{ Group C } & \multicolumn{2}{|c|}{ Total } & & \\
\hline & & $\mathbf{N}$ & $\%$ & $\mathbf{N}$ & $\%$ & $\mathbf{N}$ & $\%$ & $\mathbf{N}$ & $\%$ & $\mathrm{X}^{2}$ & P-value \\
\hline \multirow{2}{*}{ HTN } & Yes & 3 & 10.71 & 6 & 46.15 & 28 & 47.46 & 37 & 37 & \multirow{2}{*}{11.535} & \multirow{2}{*}{$\begin{array}{c}0.003^{*} \\
\mathrm{~S}\end{array}$} \\
\hline & No & 25 & 89.29 & 7 & 53.85 & 31 & 52.54 & 63 & 63 & & \\
\hline \multirow{2}{*}{ DM } & Yes & 11 & 39.29 & 6 & 46.15 & 22 & 37.29 & 39 & 39 & \multirow{2}{*}{0.353} & \multirow{2}{*}{$\begin{array}{c}0.838 \\
\text { NS }\end{array}$} \\
\hline & No & 17 & 60.71 & 7 & 53.85 & 37 & 62.71 & 61 & 61 & & \\
\hline
\end{tabular}

( $\mathrm{P}$ value for $\mathrm{HTN}=0.003$ Statistically significant). ( $\mathrm{P}$ value for $\mathrm{DM}=0.838$ Statistically non-significant). ( $\mathrm{P}$ value for $\mathrm{HTN}=0.003$ Statistically significant). ( $\mathrm{P}$ value for $\mathrm{DM}=0.838$ Statistically non-significant). In group (A), mean \pm SD of WBC was $14.637 \pm 4.861$, in group (B), mean \pm SD of WBC was $8.658 \pm 1.721$, in group $(\mathrm{C})$, mean $\pm \mathrm{SD}$ of $\mathrm{WBC}$ was $8.577 \pm 2.890$ (P value for $\mathbf{W B C}=\mathbf{0 . 0 0 1}$ Statistically significant).

In group (A), mean \pm SD of HGB was $13.239 \pm 1.689$, in group (B), mean \pm SD of HGB was $12.200 \pm 2.029$, in group $(\mathrm{C})$, mean \pm SD of HGB was $12.334 \pm 1.743$ (P value for $\mathbf{H G B}=\mathbf{0 . 0 6 4}$ Statistically non significant).

Table (6): Comparison between the studied groups regarding WBC \& HB

\begin{tabular}{|c|c|c|c|c|c|c|c|c|c|}
\hline & \multicolumn{3}{|c|}{$\mathrm{ACS}$} & \multicolumn{2}{|c|}{ ANOVA } & \multicolumn{3}{|c|}{ TUKEY'S Test } \\
\hline & & $\begin{array}{c}\text { Group } \\
\text { A }\end{array}$ & Group B & Group C & $\mathbf{F}$ & P-value & S\&NS & S\&UN & NS\&UN \\
\hline $\begin{array}{c}\text { WBC } \\
\left(\mathrm{x} 10^{9}\right. \\
/ 1)\end{array}$ & $\begin{array}{c}\text { Mean } \\
\pm \text { SD }\end{array}$ & $\begin{array}{c}14.637 \\
\pm \\
1.861\end{array}$ & $\begin{array}{c}8.658 \\
\pm \\
0.721\end{array}$ & $\begin{array}{c}8.577 \\
\pm \\
0.890\end{array}$ & 30.857 & $\begin{array}{c}<0.001 * \\
\mathrm{~S}\end{array}$ & $<0.001 *$ & $\begin{array}{c}<0.001 \\
*\end{array}$ & 0.997 \\
\hline $\begin{array}{c}\text { HB } \\
\text { (g/dl) }\end{array}$ & $\begin{array}{c}\text { Mean } \\
\pm \text { SD }\end{array}$ & $\begin{array}{c}13.239 \\
\pm \\
1.689\end{array}$ & $\begin{array}{c}12.200 \\
\pm \\
1.029\end{array}$ & $\begin{array}{c}12.334 \\
\pm \\
0.743\end{array}$ & 2.822 & $\begin{array}{c}0.064 \\
\text { NS }\end{array}$ & & & \\
\hline
\end{tabular}

( $\mathrm{P}$ value for $\mathrm{WBC}=0.001$ Statistically significant $)$. $(\mathrm{P}$ value for $\mathrm{HGB}=0.064$ Statistically non significant $)$.

In group (A), mean \pm SD of creatinine was $0.904 \pm 0.213$, in group (B), mean \pm SD of creatinine was $1.454 \pm 0.336$, in group $(\mathrm{C})$, mean \pm SD of creatinine was $1.212 \pm 0.291$ (P value for creatinine $=\mathbf{0 . 0 0 4}$ Statistically significant). 
Table (7): Comparison between the studied groups regarding creatinine.

\begin{tabular}{|c|c|c|c|c|c|c|c|c|c|}
\hline & \multicolumn{3}{|c|}{ ACS } & \multicolumn{2}{|c|}{ ANOVA } & \multicolumn{3}{|c|}{ TUKEY'S Test } \\
\hline & & Group A & Group B & $\overline{\text { Group C }}$ & $\overline{\mathbf{F}}$ & $\overline{P \text { P-value }}$ & S\&NS & S\&UN & NS\&UN \\
\hline & $\begin{array}{c}\text { Mean } \\
\pm \text { SD }\end{array}$ & $\begin{array}{c}0.904 \\
\pm \\
0.013\end{array}$ & $\begin{array}{c}1.454 \\
\pm \\
0.036\end{array}$ & $\begin{array}{c}1.212 \\
\pm \\
0.091\end{array}$ & 5.826 & $\begin{array}{c}0.004 * \\
\mathrm{~S}\end{array}$ & $0.006^{*}$ & $0.029 *$ & 0.283 \\
\hline
\end{tabular}

(P value for creatinine $=0.004$ Statistically significant $)$

In group (A), mean \pm SD of ALT was $34.429 \pm 7.829$, in group (B), mean \pm SD of ALT was $20.231 \pm 4.917$, in group (C), mean \pm SD of ALT was $20.322 \pm 4.436$ (P value for ALT=0.001 Statistically significant).

In group (A), mean \pm SD of AST was $95.429 \pm 22.111$, in group (B), mean \pm SD of AST was $30.077 \pm 6.800$, in group (C), mean \pm SD of AST was $30.780 \pm 7.484$ (P value for AST= 0.001 Statistically significant).

Table (8): Comparison between the studied groups regarding ALT \& AST.

\begin{tabular}{|c|c|c|c|c|c|c|c|c|c|}
\hline & \multicolumn{3}{|c|}{$\mathrm{ACS}$} & \multicolumn{2}{|c|}{ ANOVA } & \multicolumn{3}{|c|}{ TUKEY'S Test } \\
\hline & & Group A & Group B & Group C & $\mathbf{F}$ & P-value & S\&NS & S\&UN & NS\&UN \\
\hline $\begin{array}{l}\text { ALT } \\
(\mathrm{U} / \mathrm{L})\end{array}$ & $\begin{array}{c}\text { Mea } \\
\text { n } \\
\pm \text { SD }\end{array}$ & $\begin{array}{c}34.429 \\
\pm \\
2.829\end{array}$ & $\begin{array}{c}20.231 \\
\pm \\
1.917\end{array}$ & $\begin{array}{c}20.322 \\
\pm \\
1.436\end{array}$ & 10.872 & $\begin{array}{c}<0.001 \\
* \\
\mathrm{~S}\end{array}$ & $0.007 *$ & $<0.001^{*}$ & 1.000 \\
\hline $\begin{array}{l}\text { AST } \\
(\mathrm{U} / \mathrm{L})\end{array}$ & $\begin{array}{c}\text { Mea } \\
\mathbf{n} \\
\pm \text { SD }\end{array}$ & $\begin{array}{c}95.429 \\
\pm \\
2.111\end{array}$ & $\begin{array}{c}30.077 \\
\pm \\
2.800\end{array}$ & $\begin{array}{c}30.780 \\
\pm \\
3.484\end{array}$ & 35.569 & $\begin{array}{c}<0.001 \\
* \\
\mathrm{~S}\end{array}$ & $<0.001 *$ & $<0.001 *$ & 0.998 \\
\hline
\end{tabular}

( $P$ value for ALT $=0.001$ Statistically significant $)$, $(P$ value for AST $=0.001$ Statistically significant $)$.

In group (A), mean \pm SD of total bilirubin was $0.627 \pm 0.141$, in group (B), mean \pm SD of total bilirubin was $0.527 \pm$ 0.123 , in group $(C)$, mean \pm SD of total bilirubin was $0.795 \pm 0.188$ (P value for total bilirubin $=\mathbf{0 . 0 0 7}$ Statistically significant).

In group (A), mean \pm SD of direct bilirubin was $0.215 \pm 0.047$, in group (B), mean \pm SD of direct bilirubin was 0.126 \pm 0.026 , in group $(\mathrm{C})$, mean $\pm \mathrm{SD}$ of direct bilirubin was $0.279 \pm 0.053$ ( $\mathbf{P}$ value for direct bilirubin $=\mathbf{0 . 0 1 3}$ Statistically significant).

In group (A), mean \pm SD of indirect bilirubin was $0.412 \pm 0.102$, in group (B), mean \pm SD of indirect bilirubin was $0.401 \pm 0.100$, in group $(C)$, mean \pm SD of indirect bilirubin was $0.516 \pm 0.192$ (P value for indirect bilirubin $=\mathbf{0 . 0 0 8}$ Statistically significant).

Table (9): Comparison between the studied groups regarding total bilirubin, direct bilirubin \& indirect bilirubin.

\begin{tabular}{|c|c|c|c|c|c|c|c|c|c|}
\hline & \multicolumn{3}{|c|}{ ACS } & \multicolumn{2}{|c|}{ ANOVA } & \multicolumn{3}{|c|}{ TUKEY'S Test } \\
\hline & & Group A & Group B & Group C & $\mathbf{F}$ & P-value & S\&NS & S\&UN & NS\&UN \\
\hline $\begin{array}{c}\text { Total } \\
\text { Bilirubin } \\
\text { (mg/dl) }\end{array}$ & Mean \pm SD & $\begin{array}{c}0.627 \\
\pm \\
0.041\end{array}$ & $\begin{array}{c}0.527 \\
\pm \\
0.023\end{array}$ & $\begin{array}{c}0.795 \\
\pm \\
0.088\end{array}$ & 5.259 & $\begin{array}{c}\mathbf{0 . 0 0 7} * \\
\mathbf{S}\end{array}$ & 0.614 & 0.059 & 0.019* \\
\hline $\begin{array}{c}\text { Direct } \\
\text { Bilirubin } \\
(\mathbf{m g} / \mathbf{d l}) \\
\end{array}$ & Mean \pm SD & $\begin{array}{c}0.215 \\
\pm \\
0.047\end{array}$ & $\begin{array}{c}0.126 \\
\pm \\
0.026\end{array}$ & $\begin{array}{c}0.279 \\
\pm \\
0.053\end{array}$ & 4.580 & $\begin{array}{c}\mathbf{0 . 0 1 3}^{*} \\
\mathbf{S}\end{array}$ & 0.283 & 0.247 & 0.014* \\
\hline $\begin{array}{c}\text { Indirect } \\
\text { Bilirubin } \\
\text { (mg/dl) }\end{array}$ & Mean \pm SD & $\begin{array}{c}0.412 \\
\pm \\
0.002\end{array}$ & $\begin{array}{c}0.401 \\
\pm \\
0.010\end{array}$ & $\begin{array}{c}0.516 \\
\pm \\
0.092\end{array}$ & 5.048 & $\begin{array}{c}\mathbf{0 . 0 0 8}^{*} \\
\mathrm{~S}\end{array}$ & 0.980 & $0.021 *$ & 0.068 \\
\hline
\end{tabular}

( $\mathrm{P}$ value for total bilirubin $=0.007 *$ Statistically significant $)$. $(\mathrm{P}$ value for direct bilirubin $=0.013 *$ Statistically significant $)$, $\mathrm{P}$ value for indirect bilirubin $=0.008^{*}$ Statistically significant $)$. 
When we compared the three groups, we found that serum total bilirubin is highest in group (C), followed by group $(\mathrm{A}, \mathrm{B})$ respectively.

According to this analysis, there is an inverse correlation of bilirubin with ACS. This strengths the fact that bilirubin acts as an antioxidant and has cardioprotective action and patients with ACS have lower levels of bilirubin. So, bilirubin can be used as a factor for screening individuals who have high risk for ACS and preventive strategies applied in them before the onset of overt ACS.

\section{DISCUSSION}

This cross-sectional study included a total of 100 patients presented with acute coronary syndrome, of them $64 \%$ were men and $36 \%$ were women. The male preponderance was similar to other studies like Sahin et al. ${ }^{(15)}$ who stated that $66 \%$ of participants were males and $34 \%$ were females. Hopkins et al. ${ }^{\left({ }^{(16)}\right.}$ had $75.6 \%$ males and $24.4 \%$ females. This finding reveals the fact that the incidence of ACS in males.

Their average age was (55.4 \pm 9.622$)$ ranged from 20 to 71 years. This finding reveals the fact that the incidence of ACS increases with age which is similar to other studies like Alexander ${ }^{\left({ }^{17}\right)}$.

Total leucocyte count (TLC) in ACS is usually high as infarction of the myocardium is an inflammatory process. In our study the mean TLC was 10.284 \pm 4.375 SD, like in ${ }^{(\mathbf{1 8})}$.

Bilirubin has been long postulated to have antioxidant properties and thus its correlation with ACS is of interest ${ }^{(7)}$.

The mean bilirubin values in $\mathrm{mg} / \mathrm{dl}$ for STEMI was $0.627 \pm 0.141 \mathrm{SD}$, for Non-STEMI it was $0.527 \pm 0.173$ and for unstable angina it was $0.795 \pm$ $0.367 \mathrm{SD}$. The mean total bilirubin in patients with ACS was $0.713 \pm 0.330 \mathrm{SD} \mathrm{mg} / \mathrm{dl}$. There is statistically significance less total bilirubin of ACS than normal standard mean value $(0.6)(\mathrm{p}<0.0001)$.

The results were comparable to Sahin $\boldsymbol{e t}$ al. (15) and Hopkins et al. ${ }^{(16)}$ who also had decreased bilirubin levels in patients with ACS.

The inverse association between serum bilirubin concentrations and CAD has been found in other several studies ${ }^{(15,19,20)}$.

Sahin et al. ${ }^{(15)}$ found high serum total bilirubin level is independently associated with severity of coronary artery disease in patients with NSTEMI.

Kim et al. ${ }^{(19)}$ did cross-sectional study on 19,792 Koreans and found serum total bilirubin concentration inversely correlated with Framingham risk score and it may be helpful to decrease the future risk of Coronary artery disease.

Arumalla et al. ${ }^{(20)}$ found plasma bilirubin concentration could act as a provisional new marker of atherogenic risk that can be measured easily in the clinical laboratory and applied in medical practice as lipid oxidation and formation of oxygen radicals are important elements of arterial plaque formation and atherosclerosis, and are involved in the pathophysiology of coronary artery disease (CAD). Since bilirubin has antioxidant properties, it has been suggested that it may have a protective role in the atherosclerotic process by preventing formation of oxidized LDL. The antioxidant capacity of bilirubin and its ability to provide potent scavenging effect of peroxyl radicals have led to suggest that mild increase in the circulatory bilirubin may have a physiologic role to protect against the disease processes that involve oxygen and peroxyl radicals. This review briefly examines the role of bilirubin and its protective function against the coronary artery disease.

Also, Kharb ${ }^{(21)}$ found an inverse relationship between increase in total bilirubin and serum levels of LDL-C in myocardial infarction as they examined serum bilirubin and low density lipoprotein cholesterol (LDL-C) as possible risk factors in 27 patients of myocardial infarction and 50 healthy individuals served as control. An inverse relationship between increase in total bilirubin and serum levels of LDL-C was observed. There is possibility of bilirubin playing a role in the pathogenesis of coronary heart disease through LDL-C levels.

Giving a possibility of bilirubin playing a role in the pathogenesis of coronary heart disease through LDL-C levels. Song et al. ${ }^{(22)}$ with the objective to investigate the effects of low serum bilirubin levels on the risk for future coronary artery disease (CAD) in a prospective cohort of 8,593 subjects found the addition of low serum bilirubin levels to the traditional risk factors for $\mathrm{CAD}$, such as metabolic syndrome, may yield an improvement of risk prediction.

The same association was also reported in a recent Taiwanese prospective study by Huang $\boldsymbol{e t}$ al. ${ }^{(23)}$ on patients with cardiac $\mathrm{X}$ syndrome(which is a historic term for microvascular angina(chest pain) with signs associated with decreased blood flow to heart tissue but with normal coronary arteries) followed for 5years, in which patients with the lowest serum bilirubin levels had a higher incidence of non-fatal myocardial infarction, ischemic stroke, rehospitalization for unstable angina and coronary revascularization procedures.

Glucuronidation by UGT1A1 (UDP glucuronosyltransferase family 1 member A1) has a controlling effect on serum bilirubin levels. Because the UGT1A1*28 Gilbert syndrome polymorphism results in higher (protective) serum bilirubin levels, it is expected to be associated with reduced CVD risk. Seven studies have addressed this, and all confirmed the association of UGT1A1*28 with higher bilirubin level (24).

Among them the strongest protective effect of elevated bilirubin on cardiovascular disease was reported in a prospective population-based cohort study in the Framingham Heart Study by Lin et al. ${ }^{(24)}$ which included 1780 unrelated individuals who had been followed up for 24years found Homozygotes with 
UGT1A $1 * 28$ allele carriers with higher serum bilirubin concentrations exhibited a strong association with lower risk for cardiovascular disease.

Also in the large study by Horsfall et al. ${ }^{(25)}$ demonstrated the association between reduced serum bilirubin levels and increased CVD risk is strong in both sexes and could act as an independent risk factor. It seems likely that several factors are playing a role; antioxidant effects of bilirubin, heme oxygenase activity and consumption of bilirubin by oxidative processes could all be involved.

\section{CONCLUSIONS}

It could be concluded that there is an inverse relation of bilirubin with acute coronary syndromes (ACS). This strengths the fact that bilirubin acts as an antioxidant and has cardioprotective action and patients with ACS have lower levels of bilirubin. This could suggest bilirubin as a factor for screening individuals who have high risk for ACS and preventive strategies applied in them before the onset of overt ACS.

Our study found that hypertension, smoking and family history of IHD were significantly correlated with ACS and there is statistically significance less total bilirubin of ACS than normal standard mean value.

\section{REFERENCES}

1. Gupta R, Joshi P, Mohan V et al. (2008): Epidemiological and causation of coronary heart disease and stroke in India. Heart, 94:16-26.

2. Leeder S, Raymond S, Greenberg H et al. (2004): A race against time: the challenge of cardiovascular disease in developing economies. Columbia University New York. Available

at https://www.eldis.org/document/A18750.

3. Mackman N (2008): Triggers, targets and treatments for thrombosis. Nature, 451:914-8.

4. Fukagawa $\mathbf{N}$ (1999): Aging: is oxidative stress a marker or is it causal? Proc Soc Exp Biol Med., 222:293-8.

5. Wang $X$, Rain water $D$, Vande Berg $J$ et al. (2001): Genetic contribution to plasma antioxidant activity. Arterioscler Thromb Vasc Biol., 21:1190-5.

6. Gupta N, Singh T, Chaudhary R et al. (2016): Bilirubin in coronary artery disease: cytotoxic or protective? World J Gastrointest Pharmacol Ther., 7:469-476.

7. Wu T, Fung K, Yang C (1994): Unconjugated bilirubin inhibits the oxidation of human low density lipoprotein better than Trolox Life Sci., 54: 477-481.

8. Morita T, Imai T, Yamaguchi T et al. (2003): Induction of heme oxygenase-1 in monocytes suppresses angiotensin II-elicited chemotactic activity through inhibition of CCR2: role of bilirubin and carbon monoxide generated by the enzyme. Antioxid Redox Signal, 5:439447.

9. Kimm H, Yun J, Jo J et al. (2009): Low serum bilirubin level as an independent predictor of stroke incidence: a prospective study in Korean men and women. Stroke, 40:3422-7.
10. Erdogan T, Ciçek Y, Kocaman S et al. (2012): Increased serum bilirubin level is related to good collateral development in patients with chronic total coronary occlusion. Intern Med., 51:249-55.

11. Ajay VS, Prabhakaran D (2010): Coronary heart disease in Indians: Implications of the Interheart study. Ind J Med Res., 132(5):561-6.

12. Wilson $P$ (1994): Established risk factors and coronary artery disease: the Framingham Study. Am J Hypertens., 7(7 Pt 2):7-12.

13. Vogel B, Claessen B, Arnold S et al. (2019): ST-segment elevation myocardial infarction. Nat Rev Dis Primers, 5(1):39-43.

14. Hedayati T, Yadav N, Khanagavi J (2018): Non-STsegment acute coronary syndromes. Cardiol Clin., 36(1):37-52.

15.Sahin O, Kaya M, Akpek M et al. (2014): Relation between serum total bilirubin levels and severity of coronary artery disease in patients with non-ST-segment elevation myocardial infarction. Angiology, 65:245-9.

16. Hopkins P, Wu L, Hunt S et al. (1996): Higher serum bilirubin is associated with decreased risk for early familial coronary artery disease. Arterioscler Thromb Vasc Biol., 16:250-5.

17. Cheruvu P, Finn A, Gardner C et al. (2007): Frequency and distribution of thin-cap fibroatheroma and ruptured plaques in human coronary arteries: a pathologic study. $\mathbf{J}$ Am Coll Cardiol., 50:940-9.

18.Jan A, Habib S, Naseeb K et al. (2011): High total leukocyte count and heart failure after myocardial infarction. Pakistan Heart Journal, 44: 8-15.

19. Kim K, Kim B, Park S et al. (2012): Serum total bilirubin concentration is inversely correlated with Framingham risk score. Arch Med Res., 43:288-93.

20. Arumalla V, Gopal N, Rao B (2012): Role of serum bilirubin in coronary artery disease. Int J Med Heal Sci., 2:56-62.

21.Kharb S (2006): Association of serum concentration of total bilirubin and low-density lipoprotein cholesterol with myocardial infarction. World J Med Sci., 1:934-39.

22.Song Y, Koo B, Cho N et al. (2014): Effect of low serum total bilirubin levels $(\leq 0.32 \mathrm{mg} / \mathrm{dl})$ on risk of coronary artery disease in patients with metabolic syndrome. Am J Cardiol., 114:1695-700.

23.Huang S, Huang P, Leu H et al. (2009): Serum bilirubin predicts long-term clinical outcomes in patients with cardiac syndrome X. Heart, 96:122732.

24.Lin J, O'Donnell C, Schwaiger J et al. (2006): Association between the UGT1A1*28 Allele, Bilirubin Levels, and Coronary Heart Disease in the Framingham Heart Study. Circulation, 114:1476-81.

25.Horsfall L, Nazareth I, Petersen I (2012): Cardiovascular events as a function of serum bilirubin levelsin alarge, statin-treated cohort. Circulation, 126(22):255664. 